\title{
Estudo da relação entre variáveis fisiológicas, biomecânicas e o rendimento de corredores portugueses de 3000 metros
}

\author{
José A. Bragada \\ Tiago M. Barbosa
}

https://doi.org/10.5628/rpcd.07.03.291

\author{
D epartamento de Ciências do D esporto e Educação Física \\ Instituto Politécnico de Bragança \\ Portugal
}

\section{RESUMO}

O objectivo do presente estudo foi investigar as relações entre o perfil fisiológico, a resposta biomecânica e o rendimento desportivo de corredores de meio-fundo portugueses. Dezoito atletas foram submetidos a um protocolo incremental de 5 patamares de 3 minutos em tapete rolante sem qual quer inclinação. Em cada patamar a velocidade foi constante e os incrementos foram de 1,45 $\mathrm{km} \cdot \mathrm{h}^{-1}$. Durante todo o protocolo, o consumo de oxigénio e outros parâmetros bioenergéticos foram avaliados respiração-a-respiração através de oximetria directa. Foram calculadas a potência metabólica (Pmet), o custo energético (C), a velocidade de deslocamento ( $v$ ), a frequência gestual $(F G)$ e a distância de ciclo (DC). Foi determinado em prova oficial (corrida de 3000 metros) o rendimento desportivo. Verificou-se uma relação positiva e significativa entre a Pmet e a v $\left(R^{2}=0,78\right.$; $p<0,01)$. As relações entre o $C$ e $a F G$ ou $a D C$ não foram significativas. A relação entre a v e a $F G\left(R^{2}=0,34 ; p<0,01\right)$ e entre a v e a $D C\left(R^{2}=0,87 ; p<0,01\right)$ foram positivas e significativas. Verificou-se uma relação significativa entre o rendimento e a Pmet a $16 \mathrm{~km} \cdot \mathrm{h}^{-1}\left(R^{2}=0,23 ; p=0,05\right)$. Em conclusão, verifica-se uma dependência moderada do rendimento dos meio-fundistas portugueses relativamente ao seu perfil fisiológico e este de forma ténue face à resposta biomecânica.

Palavras-chave: Atletismo, rendimento, custo energético, distância de ciclo, frequência gestual, velocidade

\begin{abstract}
Study the relationships between physiological profile, biomechanical behaviour and performance of middle-distance Portuguese runners

The aim of this investigation was to study the relationships between physiological profile, biomechanical behaviour and performance of middle-distance Portuguese runners. Eighteen runners were submitted to an incremental protocol of 5 trials of 3 minutes in treadmill without inclination. At each step, velocity was constant and between steps velocity increased by $1,45 \mathrm{~km} \cdot \mathrm{h}^{-1}$. Through the protocol, oxygen up-take and other bioenergetical parameters were evaluated breath-by-breath with direct oximetry. It was measured the metabolic power (Pmet), the energy cost $(C)$, the running velocity $(v)$, the stride frequency $(F G)$ and the stride distance (DC). It was also recorded the performance in official competition (3.000-m event). It was verified a significant and positive relationship between $P$ met and $v\left(R^{2}=0,78 ; P<0,01\right)$. The relationships between $C$ and $F G$ or $D C$ were no-significant. The relationships between $v$ and $F G\left(R^{2}=0,34 ; p<0,01\right)$, as well as, between $v$ and $D C$ $\left(R^{2}=0,87 ; p<0,01\right)$ were statistically significant and positive. It was observed a significant relationship between performance and Pmet at $16 \mathrm{~km} \cdot \mathrm{h}^{-1}\left(R^{2}=0,23 ; p=0,05\right)$. In conclusion, for portuguese middledistance runners, it seems to exist a moderate dependence of performance from physiological profile and this one, from the biomechanical behaviour.
\end{abstract}

Key-words: running, performance, energy cost, gait length, gait fre quency, velocity 


\section{INTRODUÇÃO}

No estudo da locomoção, a avaliação do custo energético (C) é um dos parâmetros mais investigados já que representa a energia dispendida para percorrer uma determi nada unidade de distância(19, 35). Conhecendo a potência metabólica do sistema biológico (Pmet) e a velocidade de deslocamento do corpo $(v)(5)$ :

$$
C=\frac{\text { Pmet }}{V}
$$

Tem sido descrito para diversas formas de locomoção no meio terrestre, aquático e aéreo que existe uma relação entre o custo energético (C), o trabaIho mecânico total $\left(W_{\text {tot }}\right)$ e a eficiência do sistema $(\eta)^{(3,16):}$

$$
\mathrm{C}=\frac{\mathrm{W}_{\text {tot }}}{\eta}
$$

Ou seja, verifica-se o estabel ecimento de pontes entre a Mecânica Clássica com a Termodinâmica e a Bioenergética(5). Estas pontes pressupõem o entendimento do organismo biológico, como um sistema termodinâmico e o desempenho desportivo como uma emergência de processos optimizados de aporte energético, repercussão das características biomecânicas desse sistema.

Com efeito, o $\mathrm{C}$ tem sido descrito como um dos mel hores preditores da distância percorrida pela Iocomoção humana em meio terrestre e aquático(3, $19,42,43)$.

Na maioria das actividades desportivas orientadas para o rendimento, como o Atletismo, o objectivo final da sua prática é a obtenção do mel hor resultado desportivo possível em contexto competitivo. Nesta modalidade, o carácter claramente temporal e espacial do seu rendimento permite uma quantificação (bio)energética e (bio)mecânica precisa.

Portugal apresenta há al gumas décadas um número substancial de atl etas de meio-fundo e de fundo de elevado nível internacional. Nesta modalidade, a par do que acontece noutras, o rendimento desportivo é um fenómeno multifactorial. Existem vários domínios que poderão afectar a resposta fisiológica do atleta de meio fundo, como por exemplo, as determinantes de ordem biomecânica(13, 39). Esta relação está largamente descrita noutras modalidades desportivas, como é o caso da natação pura desportiva(6, 37), das actividades náuticas(32) ou da marcha(3,21). No entanto, o interesse que os atl etas de meio-fundo e fundo despertam na comunidade científica portuguesa, parece centra-se quase exclusivamente na caracterização do seu perfil fisiológi co(p.e., 9, 14, 31, 33, 34). No Atletismo, as provas de meio-fundo e fundo são tidas como actividades cíclicas cujo intuito é percorrer a distância pré-definida no menor intervalo de tempo possível. Logo, assumir uma elevada vel ocidade média de deslocamento durante a prova é um pré-requisito para aceder a um melhor rendimento desportivo. Enquanto actividade cíclica que é, mecanicamente, a velocidade média de deslocamento $(v)$ pode ser determinada com base na frequência gestual (FG) e na distância de ciclo (DC)(40):

$$
\bar{v}=F G \cdot D C
$$

Na literatura sugere-se a existência de uma relação não-linear entre a Pmet e a FG ou a DC (29, 36). Existe um valor mínimo de $F G$ e de $D C$ às quais se reporta o valor mínimo de Pmet. A diminuição ou o aumento de um dos elementos mecânicos do ciclo gestual tende a incrementar a Pmet. A parentemente, os valores mínimos da Pmet ocorrem quando os sujeitos adoptam espontânea e livremente a FG e a D C (30, 38, 39). Presumivel mente, esta auto-optimização é adquirida por intermédio de feedback interno(12, 41). Alguns motivos parecem sustentar este facto(4): (i) o aumento da DC poderá incrementar a propulsão e induzir uma mai or variação intra-cíclica da posição vertical do centro de massa do corredor; (ii) o aumento da FG promoverá incrementos do trabal ho mecânico interno devido à maior variação da posição dos centros de massa parciais do corpo tendo como referência do centro de massa total. Em todo o caso, a FG parece ser a variável que apresenta uma rel ação mais consistente com a Pmet( ${ }^{36)}$.

$O$ aumento da v é descrita como sendo induzido por mudanças características na FG e na DC e de forma individualizada(13, 28). A v mais reduzidas, o incremento decorre fundamental mente do aumento da DC, após o que este parâmetro tende a estabilizar e o contínuo aumento da $v$ decorre, desta feita, do 
aumento da $F G(18,25)$. O facto dos dois parâmetros, FG e DC serem relativamente independentes, mas relacionados com a $v$, sugere que esta última é a variável "crítica" em torno da qual a locomoção se organiza(25). Por outro lado, sugere-se que corredores de melhor nível competitivo apresentam valores de $D C$ superiores a sujeitos de nível inferior para uma mesma v (11).

A economia de corrida tem sido aceite como um critério fisiológico da eficiência e da eficácia em competição. Aliás, é considerado como um factor associado ao rendimento em provas de média e longa duração $(15,20)$ e estando correlacionadas de forma significativa(17, 24, 29). Inclusive, alguns grupos de investigação sustentam a possibilidade de ser possível predizer o rendimento, em contexto competitivo, com base em diversas características fisiológicas dos corredores, incluindo a economia da corrida(7). Foi objectivo deste trabalho estudar as hipotéticas relações entre o perfil fisiológico, a resposta biomecânica e o rendimento desportivo de corredores de meio-fundo portugueses.

\section{MATERIAL E MÉTODOS}

Amostra

Foram avaliados 18 atletas do sexo masculino $(20,06 \pm 3,24$ anos de idade, $64,33 \pm 5,93 \mathrm{~kg}$ de massa corporal; $1,74 \pm 0,05$ metros de altura; 6,06 $\pm 3,78$ anos de treino; $80,50 \pm 31,50 \mathrm{~km}$ de volume de treino semanal) especialistas em provas de meio-fundo de nacional idade portuguesa. Todos os procedimentos respeitaram a Declaração de Helsínquia para investigação com humanos.

\section{Procedimentos}

Os atletas foram submetidos a um protocolo contínuo de intensidade progressiva de 5 patamares de 3 minutos em tapete rolante (PPS 55 Sport - I,

Woodway, Alemanha) sem qualquer inclinação. Em cada patamar a velocidade foi constante e os incrementos entre patamares foram de $1,45 \mathrm{~km} \cdot \mathrm{h}^{-1}(0,40$ $\left.\mathrm{m} \cdot \mathrm{s}^{-1}\right)$. A escolha dos patamares foi realizada individualmente para cada corredor, no sentido da velocidade do último coincide-se com a velocidade a que ocorre uma concentração de $4 \mathrm{mmol} \cdot \mathrm{L}^{-1}$ de lactato sanguíneo. Para a totalidade da amostra foram avaliados 90 patamares ( 18 corredores $\times 5$ patamares).
Recolha dados

Ao longo de todo o protocolo, o consumo de oxigénio e outros parâmetros bioenergéticos foram avaliados respiração-a-respiração, através de oximetria directa, utilizando um analisador de gases portátil (M etalyzer 3B, Cortex Biophysik, Alemanha). A potência metabólica (Pmet) foi expressa com base no consumo de oxigénio líquido ( $\mathrm{VO}_{2}$ net) no último minuto de cada patamar. O cálculo da Pmet foi efectuado de acordo com os procedimentos descritos por Daniels e Daniels(17) e por Brisswalter e Legros (10). Foi calculado o valor individual da $P$ met à velocidade de $16 \mathrm{~km} \cdot \mathrm{h}^{-1}\left(4,44 \mathrm{~m} \cdot \mathrm{s}^{-1}\right)$, por meio de interpolação ou extrapolação dos valores da recta de regressão individual. $\mathrm{O} C$ foi calculado com recurso à equação(1). O C foi convertido em unidades SI sabendo que $1 \mathrm{mlO}_{2}$ equivale a 20,1)(27).

A v, a FG e a DC foram calculadas no último minuto de cada patamar. A $v$ real do ergómetro foi determinada conhecendo o perímetro do tapete rolante $(\mathrm{P})$, no caso 3,42 m, e o intervalo de tempo (t) (13) para efectuar 25 revoluções:

$$
\bar{v}=\frac{25 \cdot P}{v}
$$

A FG foi determinada avaliando o intervalo de tempo (t) necessário para o sujeito efectuar 25 ciclos gestuais completos:

$$
F G=\frac{t}{25}
$$

A DC foi calculada conhecendo a v e a FG, tal como descrito na equação (3).

O rendimento desportivo foi determinado em prova oficial de meio fundo (corrida de 3000 metros) com um intervalo nunca superior a uma semana (précompetitiva ou pós-competitiva) à aplicação do protocolo.

Procedimentos estatísticos

Após a verificação das propriedades dos estimadores dos mínimos quadrados, foram comparados modelos de regressão linear e não linear. Dado que em todas as circunstâncias os modelos lineares apresentaram um Coeficiente de Determinação mais elevado e um erro da estimativa inferior, serão apresentados os modelos de regressão linear simples: (i) entre as variáveis fisio- 
lógicas (Pmet e C) e as variáveis biomecânicas ( $\mathrm{V}$, FG e $D C$ ); (ii) entre a v e os parâmetros mecânicos do ciclo gestual (FG e DC); (iii) individuais para interpolação e/ ou extrapolação da Pmet a uma dada v; (iv) entre a Pmet a dada v e o rendimento desportivo. $O$ nível de significância foi definido em $p \leq 0,05$. A análise estatística (SPSS, versão 9.0, Chicago, Illinois, EUA) e a produção dos gráficos (StatView, versão 4.53, Berkley, Califórnia, EUA) foram efectuadas em programas informáticos específicos.

\section{RESULTADOS}

A figura 1 apresenta a rel ação entre a P met, definida a partir do $\mathrm{VO}_{2}$ net, e a velocidade média de deslocamento. Verificou-se uma relação positiva, el evada e significativa $\left(R^{2}=0,78 ; p<0,01\right)$ onde $o$ incremento da $v$ promoveu um aumento da Pmet.

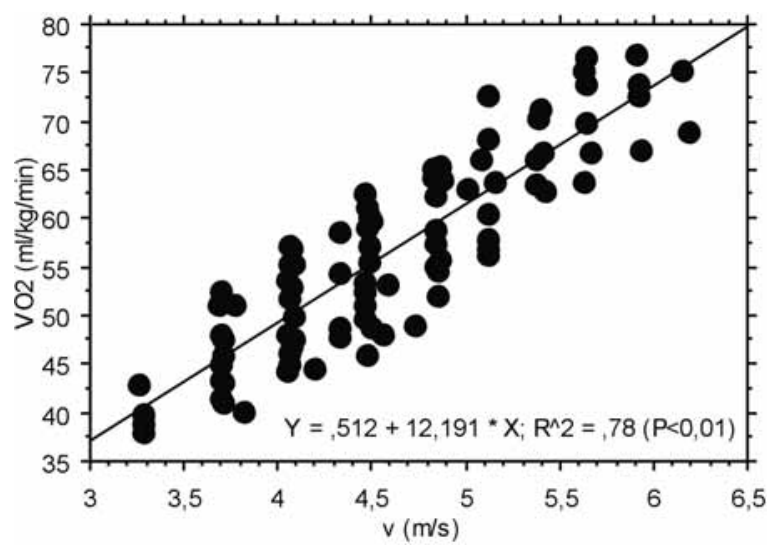

Figura 1. Relação entre o consumo de oxigénio $\left(\mathrm{VO}_{2}\right)$ e a velocidade de deslocamento $(\mathrm{V})$

A figura 2 e 3, respectivamente, apresentam a relação entre o $C$ e os parâmetros mecânicos do ciclo gestual. Em ambos os casos, as relações entre a variável bioenergética e as variáveis biomecânicas não apresentaram valores estatisticamente significativos.

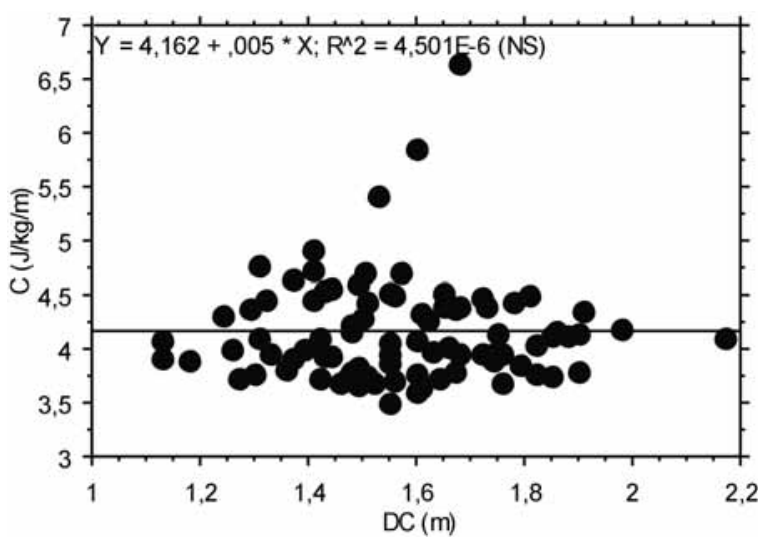

Figura 2. Relação entre o custo energético (C) e a distância de ciclo (DC).

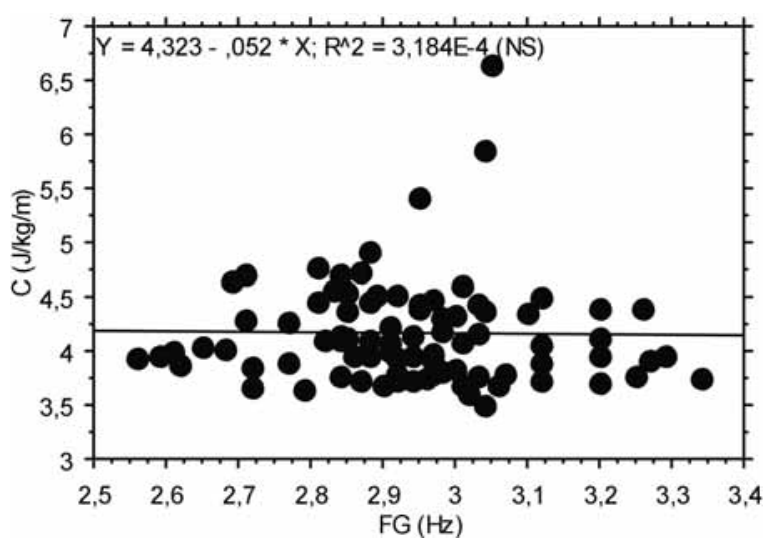

Figura 3. Relação entre o custo energético (C) e a frequência gestual (FG).

As figuras 4 e 5 apresentam as relações verificadas entre as três variáveis mecânicas do ciclo gestual adoptadas. Observaram-se resultados significativos quer para a relação entre a v e a $F G\left(R^{2}=0,34\right.$; $p<0,01)$, quer para a relação entre a v e a $D C$ $\left(R^{2}=0,87 ; p<0,01\right)$. Nesta circunstância, aumentos da v parecem decorrer de incrementos da FG, mas com maior consistência da DC . 


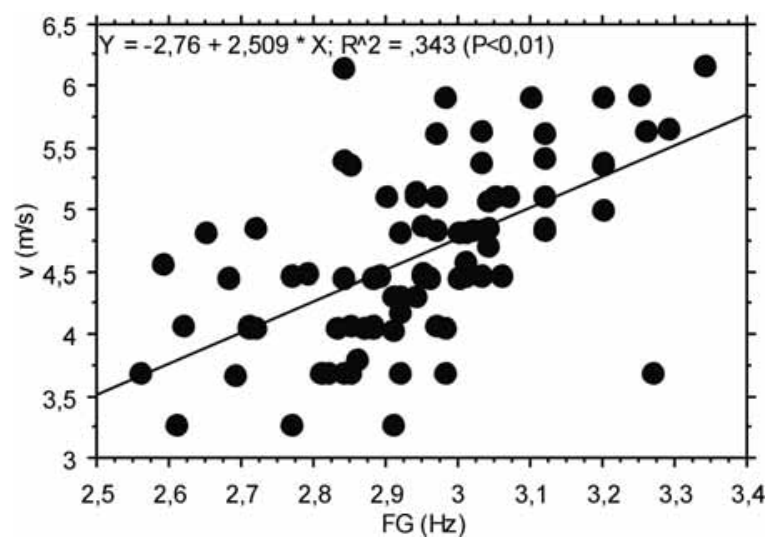

Figura 4. Relação entre a velocidade de deslocamento ( $v$ ) e a frequência gestual (FG).

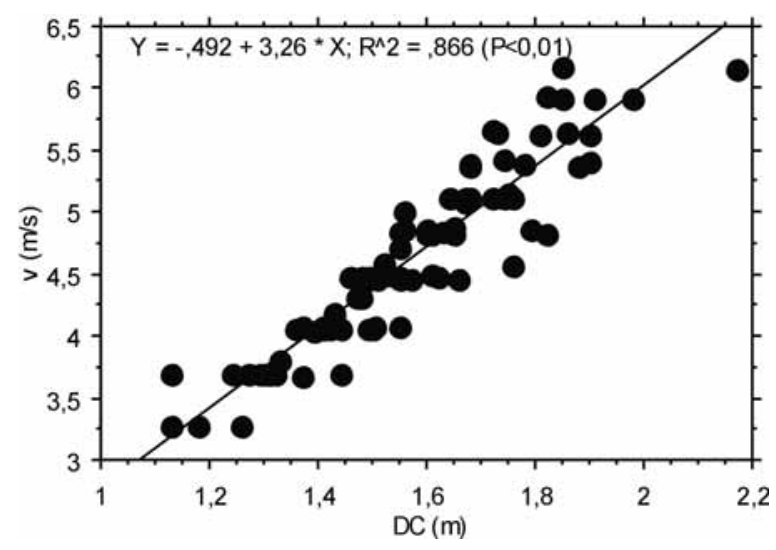

Figura 5. Relação entre a velocidade de deslocamento (v) e a distância de ciclo (DC).

A figura 6 apresenta a relação entre o rendimento desportivo dos atletas portugueses de meio-fundo, em prova de 3000 metros e a economia de corrida, definida através da interpol ação e/ ou extrapolação dos valores de $P$ met das rectas individuais para a velocidade de $16 \mathrm{~km} \cdot \mathrm{h}^{-1}$. Observou-se uma relação moderada, mas significativa onde o mel hor rendimento estava relacionado com um menor dispêndio energético à velocidade definida $\left(R^{2}=0,23 ; p=0,05\right)$. Isto é, para a amostra estudada, $23 \%$ do rendimento na prova de 3000 metros foi explicada pela economia de corrida dos atletas.

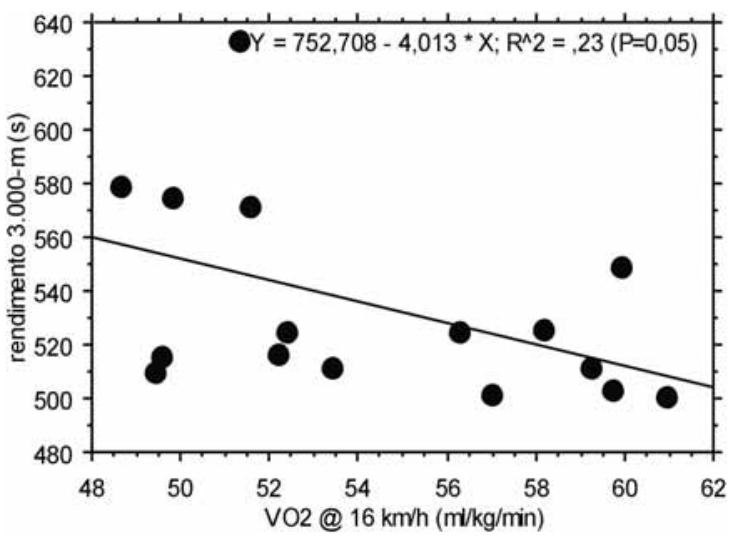

Figura 6. Relação entre o rendimento desportivo em prova de 3000 metros e a economia de corrida.

\section{DISCUSSÃO}

O objectivo do presente estudo foi investigar as relações entre o perfil fisiológico, a resposta biomecânica e o rendimento desportivo de corredores de meiofundo portugueses. Verificou-se uma dependência moderada do rendimento dos meio-fundistas relativamente ao seu perfil fisiológico e este de forma ténue face à resposta biomecânica.

No que se concerne à relação entre variáveis bioenergéticas e biomecânicas, observou-se uma relação positiva e significativa entre a Pmet e a v, tal como é frequentemente descrito na literatura (16, 23, 26). Já as relações entre o C e a FG ou a DC não foram estatisticamente significativas.

Tem sido descrita uma relação não linear entre a Pmet com a FG e a DC onde a diminuição ou o aumento de um dos elementos mecânicos do ciclo gestual tende a incrementar a variável bioenergética(29, 36). Sugere-se uma explicação deste fenómeno com base em diversos processos de controlo motor. A FG é modulada por um mecanismo particular assumido pelo sistema nervoso central(44). A mudança na frequência segmentar está associada com mudanças de rigidez global dos segmentos em fase não propulsiva, sugerindo que a FG é alterada pela mudança da actividade tónica dos músculos da cadeia cinemática em fase não propulsiva(8). O sistema nervoso central tenderá a modular a coactivação dos músculos antagonistas durante a manipulação da FG. Sabendo que a coactivação é metabolicamente dispendiosa, o aumento da FG irá repercutir-se 
aumentando o C. Já Anderson(4) defende que a variação da DC poderá induzir uma maior variação intracíclica da posição vertical do centro de massa do corredor e consequentemente $\mathrm{o} C$.

Outro mecanismo explicativo da minimização do $C$ é considerar a locomoção humana a partir de modelo do pêndulo. Assumindo toda a massa do sujeito concentrada no seu centro de massa total e os seus membros inferiores como segmentos rígidos de massa negligenciável, a velocidade do centro de massa é definida através de uma função sinusoidal. Ao longo do ciclo gestual, essa variação intra-cíclica da velocidade é explicada pela transferência sucessiva de energia cinética e de energia potencial gravítica ao longo do ciclo gestual(1). Já num modelo matemático mais realístico, assume-se que igualmente, o armazenamento e recuperação de energia mecânica em estruturas elásticas al ongadas, tal como em molas, terá um efeito determinante na minimização do $C(1,26)$.

Contudo, o presente estudo não identificou relações significativas entre as variáveis em discussão. A explicação será: (i) a amplitude de velocidades estudadas ter sido mais reduzida do que as dos autores supra-citados, o que matematicamente dificulta o ajuste de uma função matemática do tipo proposta por eles e; (ii) se ter optado pelo estudo da relação do $C$ com os parâmetros biomecânicos, o que já elimina o efeito multicolinear da v. Kraemer e Eck(23) acrescentam um outro motivo para a ausência de relação. A v superiores ou inferiores à auto-sel ecionada, ocorrerá uma alteração na cinemática angular dos principais centros articulares do membro inferior com repercussões no momento de inércia, bem como, na oscilação vertical do centro de massa e, portanto, no $\mathrm{C}$.

Curiosamente, ao efectuar uma análise individual dos corredores da amostra verificou-se que cada um deles apresentava uma estratégia individual de maximização da sua eficiência ao longo do protocolo. Diversos atletas apresentaram diminuições do $C$ com o aumento da FG e estabilização ou ligeira diminuição da DC e outros a estratégia oposta. Isto significará que o constrangimento a uma auto-selecção, eminentemente individual, da estratégia técnica a adoptar terá como repercussão não uma diminuição do $C$ mas antes no seu aumento(21).
Diversas técnicas de locomoção terrestre foram descritas como apresentando uma relação polinomial entre a v e a FG ou a DC (p.e., 22, 26, 40). Contudo, no presente estudo verificou-se um maior ajuste e um menor erro de estimativa ao adoptarem-se modelos lineares. Presumivelmente, a menor amplitude de $v$ estudadas do que a literatura, teve como consequência um maior ajuste de funções lineares.

Relativamente à relação entre as variáveis mecânicas do ciclo gestual, a relação entre a v e a FG, assim como, entre a v e a DC foram positivas, significativas e, respectivamente, modera e elevada. $\mathrm{O}$ facto da determinação da v face à $F G$ e à $D C$ apresentarem graus diferentes (moderada e el evada) confirma o facto dos dois parâmetros, serem relativamente independentes, mas relacionados com a v. Isto sugere que a v é a variável "crítica" em torno da qual a locomoção se organiza(25). Diversos estudos já tinham descrito estratégias individual izadas dos sujeitos para aumentar a v através de incremento ora da FG, ora da $D C(18,25)$. Com efeito, este é um fenómeno que extravasa a realidade da espécie humana, tendo sido também descrita para outras espécies que se deslocam pontualmente na posição bípede(2).

De forma ténue, sustenta-se que é possível associar e predizer o rendimento em provas de meio-fundo e fundo com base na economia de corrida(7, 17, 24, 29). No presente estudo, observou-se uma relação moderada, mas significativa onde o melhor rendimento estava relacionado com um menor dispêndio energé tico à velocidade definida. Para a amostra estudada, $23 \%$ do rendimento na prova de 3000 metros foi explicada pela economia de corrida dos atletas. Contudo, sendo o rendimento desportivo um fenómeno multifactorial, não deixa de ser interessante constatar que os parâmetros bionergéticos têm uma capacidade preditiva da locomoção humana em meio terrestre satisfatória(3, 19, 42).

Em conclusão, verificou-se uma relação determinística entre as variáveis estudadas, onde: (i) os parâmetros mecânicos do ciclo gestual ( $F G$ e DC) foram preditores respectivamente moderado e elevado da v; (ii) a v foi uma elevada preditora da Pmet e; (iii) a economia de corrida foi uma moderada preditora do rendimento desportivo. Ou seja, genericamente, verificou-se uma dependência moderada do rendimento dos meio-fundistas portugueses relativamen- 
te ao seu perfil fisiológico e este de forma elevada face à resposta biomecânica. Assim sugere-se que, quer treinadores, quer atletas, passem a dar uma maior atenção ao padrão técnico de corrida já que nele poderá residir o alicerce para um melhor rendimento desportivo.

\section{CORRESPONDÊNCIA}

\section{Tiago M. Barbosa}

Instituto Politécnico de Bragança

Departamento de Ciências do Desporto

e Educação Física

Campus de Sta. Apolónia

A partado 1101

5301-856 Bragança

Portugal

E-mail: barbosa@ipb.pt

\section{REFERÈNCIAS}

1. Alexander MCN (2003). Modelling approaches in biomechanics. Phil Trans R Soc Lond B 358: 1429-1435

2. Alexander MCN (2004). Bipedal animals, and their differences from humans. J A nat 204: 321-330

3. Alexander MCN (2005). Models and the scaling of energy costs for locomotion. J Exp Biol 208: 1645-1652

4. Anderson T (1996). Biomechanics and running economy. Sports M ed 22: 76-89

5. Barbosa TM, Vilas-Boas JP (2005). Estudo de diversos conceitos de eficiência da locomoção humana no meio aquático. Rev Port Ciên Desp 3: 337-349

6. Barbosa TM, Lima F, Portela A, Novais D, Machado L, Colaço P, Gonçalves P, Fernandes RJ, Keskinen KL, VilasBoas JP (2006). Relationships between energy cost, swimming velocity and speed fluctuation in competitive swimming strokes. Rev Port Ciên D esp 6(supl 2): 192-194

7. Billat V, Demarle A, Paiva M, Koralsztein J (2002). Effect of training on the physiological factors of performance in elite marathon runners (males and females). Int J Sports M ed 23: 336-341

8. Bonnard M, Paihous J (1993). Intentionality in human gait control: modifying the frequency-to-amplitude relationship. J Exp Psychol: Hum Percept and Performance 19: 429443

9. Bragada J (2003). Estudo longitudinal do rendimento e de parâmetros da carga (interna e externa) em corredores de 3000m. Tese de Doutoramento. Porto: Faculdade de Ciências do Desporto e de Educação Física da Universidade do Porto

10. Brisswalter J, Legros P (1994). Daily stability in energy cost of running, respiratory parameters and stride rate among well-trained middle distance runners. Int J Sports M ed 15: 238-241

11. Cavanagh P, Williams K (1982). The effect of stride length variation on oxygen uptake during distance running. $M$ ed Sci Sports Exerc 14: 30-35

12. Cavanagh P, Kram R (1985). The efficiency of human movement - a statement of the problem. M ed Sci Sports Exerc 17: 304-308

13. Cavanagh P, Kram R (1990). Stride length in distance running: velocity, body dimensions and added mass effects. In Cavanagh $\mathrm{P}$ (ed.). Biomechanics of Distance Running. Champaign, Illinois: Human Kinetics Books, 35-63

14. Colaço $P$ (2007). Avaliação da prestação aeróbia e anaeróbia em corredores de meio-fundo e fundo. Tese de Doutoramento. Porto: Faculdade de Ciências do Desporto e de Educação Física da Universidade do Porto

15. Cunningham $L$ (1990). Relationship of running economy, ventilatory threshold and maximal oxygen consumption to running performance in high school females. Res Q Exerc Sports 61: 369-379

16. Daniels J (1985). A physiologist's view of running economy. Med Sci Sports Exerc 17: 1-23

17. Daniels J, Daniels N (1994). Running economy of elite male and elite female runners. M ed Sci Sports Exerc 24: 369-374

18. de Luhtanen P, Komi $P$ (1978). Mechanical factors influencing running speed. In: Asmussen $E$, Jorgensen $K$ (eds.). Biomechanics VI-B. Baltimore: University Park Press, 23-29

19. di Prampero PE (1986). The energy cost of human locomotion on land and in water. Int J Sports M ed 7: 55-72 
20. Grant S, Craig I, Wilson J, Aitchison T (1997). The relationship between $3 \mathrm{~km}$ running performance and selected physiological variables. J Sports Sci 15: 403-410

21. Gutmann A, Jacobi B, Butcher T, Bertram J (2006). Constrained optimization in human running. J Exp Biol 209: 622-632

22. Hay J (2002). Cycle rate, length and speed of progression in human locomotion. J A ppl Biomech 18: 257-270

23. Kramer P, Eck G (2000). Locomotor energetics and leg length in hominid bidepality. J H um Evo 38: 651-666

24. Krahenbuhl G, Morgan D, Pangrazi R (1989). Longitudinal changes in distance-running performance of young males. Int I Sports M ed 10: 92-96

25. Laurent M, Pailhous J (1986). A note on modulation of gait in man: effects of constraining stride length and frequency. Hum M ov Sci 5: 333-343

26. Minetti A, Alexander MCN (1997). A theory of metabolic costs for bipedal gaits. J Theor Biol 186: 467-476

27. Mietti A (1998). The biomechanics of skipping gaits: a third locomotion? Proceedings of the R oyal Society of London B265:1227-1235

28. Minetti $A$ (2000). The three modes of terrestrial locomotion. In: Nigg B, Maclntosh B, Mester J (eds.). Biomechanics and Biology of M ovement. Champaign, Illinois: Human Kinetics, 67-78.

29. Morgan D, Baldini F, Martin P, Kohrt W (1989). Ten kilometre performance and predicted velocity at $\mathrm{VO} 2 \mathrm{max}$ among well-trained male runners. M ed Sci Sports Exerc 21: 78-83

30. Morgan D, Pate R (2004). Could the correlation between maximal oxygen uptake and "economy" be spurious? M ed Sci Sports Exerc 36: 345

31. Paiva M (2003). Relação entre a performance na maratona a partir da relação lactato-velocidade obtida num teste de terreno. Tese de Doutoramento. Porto: Faculdade de Ciências do Desporto e de Educação Física da Universidade do Porto.

32. Pendergast $D$, Zamparo $P$, di Prampero PE, Capelli $C$, Cerrettelli P, Termin A, Craig AB, Bushnell D, Paschke D, Mellendorf J (2003). Energy balance of human locomotion in water. Eur J A ppl Physiol 90: 377-386
33. Proença J (1990). Potencial informativo da concentração plasmática de lactato na condução do processo de treino. Tese de Doutoramento. Lisboa: Faculdade de Motricidade Humana da Universidade Técnica de Lisboa.

34. Santos P (1995). Controlo do treino em corredores de meio fundo e fundo. Tese de Doutoramento. Porto: Faculdade de Ciências do Desporto e de Educação Física da Universidade do Porto.

35. Schmidt-Nielsen K (1972). Locomotion: energy cost of swimming, flying and running. Science 177: 222-228

36. Sparrow W, Hughes K, Russel A, Rossignol P (2000). Movement economy, preferred modes and pacing. In: Sparrow W (ed.). Energetics of H uman A ctivity. Champaign, Illinois: Human Kinetics, 96-123

37. Wakayoshi K, D'Acquisto J, Cappaert JM, Troup JP (1995). Relationship between oxygen uptake, stroke rate and swimming velocity in competitive swimming. Int J Sports M ed 16: 19-23

38. Williams K, Cavanagh P (1987). Relationship between distance running mechanics, running economy and performance. J A ppl Physiol 63: 1236-1246

39. Williams K (1990). Relationships between distance running biomechanics and running economy. In: Cavanagh $P$ (ed.). Biomechanics of Distance Running. Champaign, Illinois: Human Kinetics Books, 271-305

40. Williams K (2000). The dynamic of running. In: Zatsiorsky V (ed.). Biomechanics in Sport. Oxford: Blackwell Science, 161-183

41. Wit B, Clecq D, Aerts P (2000). Biomechanical analysis of the stance phase during barefoot and shod running. J Biomech 33: 269-278

42. Zamparo P, Perini R, Orizio C, Sacher M, Ferretti G (1992). The energy cost of walking or running on sand. Eur J A ppl Physiol Occup Physiol 65: 183-187

43. Zamparo P, Pendergast D, Mollendorf J, Termin A, Minetti A (2005). An energy balance of front crawl. Eur J A ppl Physiol 94: 134-144

44. Zijlstra W, Rutgers A, Holf A, van Weerden T (1995). Voluntary and involuntary adaptation of walking to temporal and spatial constraints. Gait Posture 3: 13-18 